\title{
LA FLORE VASCULAIRE DES MONTS DES TRARA (NORD OUEST ALGÉRIEN)
}

\author{
Boumediene MEDJAHDI*1, Mohammed IBN TATTOU², \\ Djamel BARKAT ${ }^{1} \&$ Khéloufi BENABEDLI ${ }^{3}$ \\ ${ }^{1}$ Département de Foresterie, Faculté des Sciences, Université de Tlemcen \\ ${ }^{2}$ Institut Scientifique, BP. 703, Avenue Ibn Battouta, Rabat-Agdal \\ ${ }^{3}$ Département de Biologie, Université de Mascara, Algérie \\ *Corresponding author: zoinif@yahoo.f
}

Recibido el 27 de enero de 2009, aceptado para su publicación el 12 de febrero de 2009

Publicado "on line" en marzo de 2009

\begin{abstract}
RÉSUMÉ. La flore vasculaire des monts des Trara (Nord Ouest Algérien). Les monts des Trara, important massif du littoral oranais (Nord Ouest algérien), offre des conditions particulièrement favorables pour le développement d'une végétation luxuriante riche en taxons endémiques et/ou rares. L'analyse de la flore vasculaire forestière des Trara a révélé l'existence de 558 taxons appartenant à 87 familles et 306 genres. Un nombre important d'endémiques a été inventorié: 37 algéro-marocains, 41 ibéro-algéro-marocains, 17 sont endémiques d'Afrique du Nord (Algérie, Maroc, Tunisie) et 15 sont endémiques d'Afrique du Nord et de la Péninsule ibérique. Une dizaine de taxons rares et endémiques ont été proposés pour faire l'objet d'un programme de conservation en même temps que la création d'une réserve naturelle.
\end{abstract}

Mots clés. Inventaire, endémisme, Conservation, Monts des Trara, Nord Ouest Algérien.

SUMMARY. The vascular flora of the Trara mounts (North Western Algerian). Trara mounts is important clump of oranian coast (North-western Algerian). It provide a particularly favorable conditions for development of a rich luxurious vegetation with endemic taxa and/or rare. The analysis of the forest vascular flora in Trara has revealed the existence of 558 taxa which belong to 87 families and 306 genera. An important endemics number has recorded: 37 algero-moroccan, 41 ibero-algero-moroccan, 17 are endemic in North Africa (Algeria, Morocco, Tunisia) and 15 are endemic in Iberian peninsula and North Africa. About ten rare endemics taxa has been proposed to be involved in conservation measures including creation of a natural reserve.

Key word. Inventory, endemics, conservation, Trara mounts, North-Western Algerian.

INTRODUCTION

Le présent travail entre dans le cadre d'une thèse d'Etat sur les monts des Trara. Le catalogue établi est le résultat de compagnes floristiques des années 2005, 2006, 2007 et 2008. Il faut rappeler que ce type d'étude fait défaut en Algérie. Depuis la parution de la Flore 
de Quézel et Santa (1962), aucune actualisation n'a été réalisée. Si à l'état actuel, des mises à jour restent difficiles à mettre en œuvre à l'échelle nationale, les travaux au niveau régional (dans notre cas les Trara) par contre sont plus simples.

\section{Présentation de la zone d'étude}

Les monts des Trara se situent sur la bordure Sud occidentale du bassin méditerranéen. Ils appartiennent aux chaînes littorales de l'Oranie (Fig. 1). Ces montagnes sont soumises à un climat méditerranéen semi-aride chaud avec une moyenne des précipitations annuelles atteignant $306,80 \mathrm{~mm}$ et une température moyenne de $18,59^{\circ} \mathrm{C}$. Ce méso-climat est loin de régner sur l'ensemble des Trara. L'orographie de la région est très caractéristique, avec un allongement parallèle à la côte des principaux reliefs, formant ainsi des barrières relativement continues, sur le trajet des masses d'air venant de la mer, donnant naissance à des conditions topographiques très favorables pour capter l'humidité atmosphérique. Cette caractéristique est aussi avantagée par la disposition du réseau hydrographique (Medjahdi, 2001).

La partie littorale de l'Algérie est la plus instable et la plus diversifiée en substrats géologiques. Les strates géologiques appartiennent aux quatre ères: du primaire au quaternaire.

Le jeu de la lithologie, du relief et du climat permet une stratification écologique, par conséquent une diversité floristique et faunistique originale pour la région (Medjahdi, 2001).

\section{Végétation naturelle}

Juste après les ceintures de végétation psammo-halophile et halophile, s'installe une végétation ligneuse non halophile, souvent dominée par des espèces arborescentes. En effet, les conditions écologiques de cette zone littorale, sont propices pour le chêne liège, très sporadique sur les substrats acides. C'est également là, mais plus prés de la mer que se trouvent les formations à genévrier oxycèdre et genévrier rouge qui restent tous deux liés

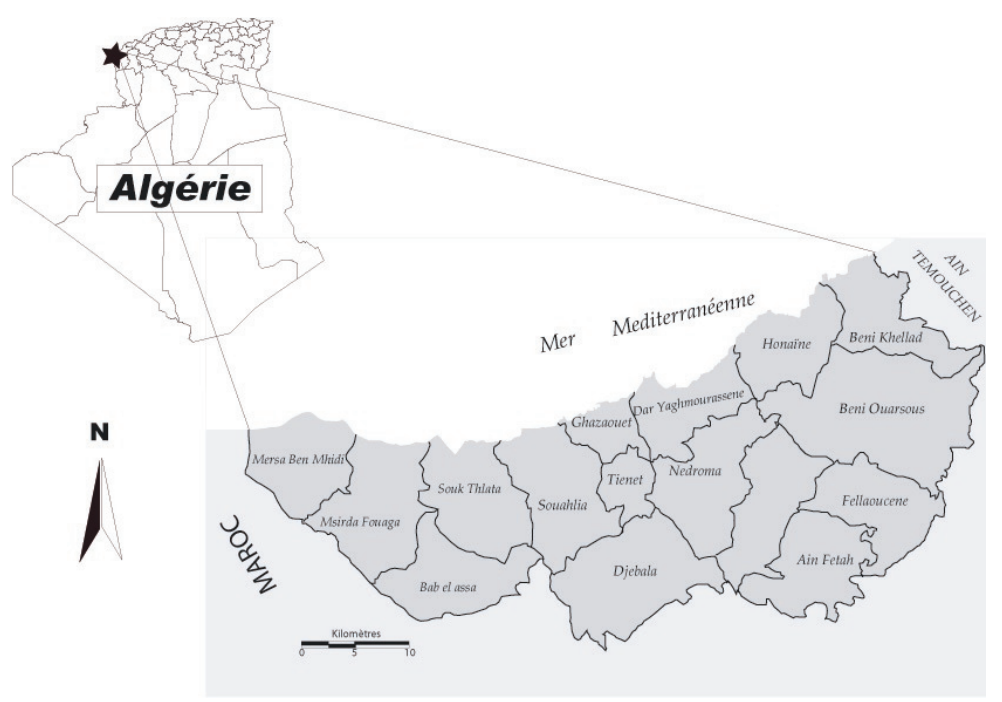

Figure 1. Localisation de la zone d'étude (Medjahdi et al, 2008) 
aux substrats sableux des dunes (Meziani et Belgats, 1984).

En dehors de ces espèces qui restent localisées, le thuya est le plus répandu dans ces zones maritimes. Grâce à sa capacité de s'accommoder de différents substrats, il se mélange aux autres essences; néanmoins, la tétraclinaie pure apparaît également en plusieurs endroits sur le littoral (Hadjadj, 1995).

Le pin d'Alep est aussi présent, mais il est partout "artificiel sinon subspontané" (Alcaraz, 1982) et ses reboisements réussissent bien.

\section{MATERIEL ET METHODES}

La réalisation de ce catalogue à été faite à partir de travaux de terrain au cours des années 2005, 2006, 2007 et 2008 qui nous ont permis de constituer un herbier. Des données bibliographiques ont été également exploitées (Hadjadj, 1995). Les échantillons témoins sont déposés dans un herbier personnel dans l'attente d'une prise en charge officielle. Les travaux de base utilisés pour l'identification des taxons recueillis sur le terrain, sont les Flores de l'Algérie et du Maroc (Quézel et Santa (1962), Fennane et al. (1999 \& 2007) et Valdes et al. (2001)).

Les informations données dans ce catalogue se présentent dans l'ordre suivant:

Nom scientifique: La nomenclature adoptée est celle de la flore d'Algérie ou à défaut de la Flore pratique du Maroc (Fennane et al. (eds.), 1999) et du Catalogue des Plantes vasculaires du Nord du Maroc de Valdés et al. (eds.) (2001).

Type biologique: $\mathrm{Ph}$. (Phanérophyte), Nph. (Nanophanérophyte), Ch. (Chaméphyte), H. (Hémicryptophyte), G. (Géophyte), Th. (Thérophyte), Phl. (Phanérophytes liane).

Répartition géographique: Pour chaque taxon la répartition biogéographique en Algérie est indiquée pour les divisions adoptées par Quézel et Santa (1962):

01: Sahels littoraux oranais 02: plaines littorales oranaises. O3: 1'Atlas tellien oranais. A1: littoral algérois. A2: 1'Atlas tellien algérois. K1: la Grande Kabylie K2: la Petite Kabylie. K3: la Numidie. C1: Tell constantinois. H1: Hauts-plateaux algérois et oranais. H2: Hautsplateaux constantinois. Hd: Hodna. AS1: 1'Atlas saharien oranais. AS2: 1'Atlas saharien algérois. AS3: 1'Atlas saharien constantinois. SS1: Sahara septentrional occidental. SS2: Sahara septentrional oriental. SO: Sahara Occidental. SC: Sahara Central. SM : Sahara méridional.

Espèces endémiques: On a indiqué pour les taxons endémiques la catégorie d'endémisme. Nous distinguons ainsi les endémiques de l'Algérie et du Maroc (AM); de l'Algérie, du Maroc et de la Péninsule Ibérique (AMI); de l'Algérie, du Maroc et de la Tunisie (Afn) et enfin de l'Afrique du Nord et de la Péninsule Ibérique (AfnI).

Espèces rares: Afin d'avoir une idée sur l'abondance globale de chaque espèce échantillonnée dans la région étudiée, nous avons adopté une échelle de trois niveaux (C): pour les taxons communs, (AC): pour les taxons peu communs $(\mathbf{R})$ : pour les taxons très rares (observés qu'une seule fois). Ce degré est déterminé à partir de la fréquence de chaque taxon dans les 250 relevés (réalisés dans le cadre de notre travail de thèse).

\section{RÉSULTATS}

PTERIDOPHYTES

ADIANTACEAE

Adiantum capillus-veneris L. G., (O1-2-3, A1-2, K1-2-3, C1 et AS1-2-3), C. 


\section{ASPLENIACEAE}

Asplenium Adiantum-nigrum L. G., (O1-2-3, A1-2, $\mathrm{K} 1-2-3, \mathrm{C} 1$ et AS1-2-3), AC.

Asplenium ceterach L. G., (O1-2-3, A1-2, K1-2-3, C1 et AS1-2-3), AC.

Asplenium hemionitis L. G., (A1), R.

Asplenium obovatum Viv. G., (K 1-2-3, C1 et O1), R.

Asplenium trichomanes L. G., (O1-2-3, A1-2, K12-3, C1 et AS1-2-3), AC.

\section{EQUISETACEAE}

Equisetum ramosissimum Desf. G.(NPh),, (O1-2-3, A1-2, K1-2-3, C1, H1-2, Hd et AS1-2-3), AC.

\section{ISOETACEAE}

Isoetes duriaei Bory G., (K1, A1-2 et O1), R.

Isoetes hystrix Bory G., (O1-2-3, A1-2, K1-2-3 et C1), AC.

\section{POLYPODIACEAE}

Polypodium cambricum L. G., (O1-2-3, A1-2, K12-3 et $\mathrm{C} 1$ ), AC.

\section{SELAGINALLACEAE}

Selaginella denticulata (L.) Spring H., (O1-2-3, A1-2, K1-2-3 et C1), C.

\section{SINOPTERIDACEAE}

Cheilanthes guanchica Bolle H., (localité non disponible), AC.

Cheilanthes maderensis Lowe H., (localité non disponible), R.

Cosentinia vellea (Aiton) Tod. H., (O1-2-3, A1-2, K1-2-3, C1 et AS1-2-3), AC.

\section{GYMOSPERMES}

CUPRESSACEAE

Juniperus oxycedrus L. Ph., (O1-2-3, A1-2, K1-2-3, $\mathrm{C} 1, \mathrm{H} 1-2, \mathrm{Hd}$ et AS1-2-3) AC.

Juniperus phoenicea L. Ph., (O1-2-3, A1-2, K1-2-3, H1-2 et AS1-2-3) C.

Tetraclinis articulata (Vahl.) Masters Ph., (O1-2-3,
A1-2, H1 et K1) C.

EPHEDRACEAE

Ephedra altissima Desf. Phl., (O1-2-3, A1-2, K12-3, C1, H1-2, Hd et AS1-2-3) C.

Ephedra fragilis Desf. Phl., (O1-2-3, A1-2, K1-2-3 et $\mathrm{C} 1$ ), AC.

\section{PINACEAE}

Pinus halepensis Mill. Ph., (O1-2-3, A1-2, K1-2-3, C1, H1-2, Hd et AS1-2-3), C.

\section{DICOTYLEDONES}

ACANTHACEAE

Acanthus mollis L. H. (O1-2-3, A1-2, K1-2-3, C1), AC.

\section{AIZOACEAE}

Mesenbryanthemum crystallinum L. Th., (O1), C.

\section{ANACARDIACEAE}

Pistacia lentiscus L. Nph., (O1-2-3, A1-2, K1-2-3, C1, H1-2, Hd et AS1-2-3), C.

Rhus pentaphylla Desf. Nph., (O1-2-3 et K3), $\mathrm{AC}$.

\section{APOCYNACEAE}

Nerium oleander L. Ph., (O1-2-3, A1-2, K1-2-3, C1, H1-2, Hd et AS1-2-3), C.

\section{ARISTOLOCHIACEAE}

Aristolochia baetica L. Phl., (O1-2-3), [AMI], C.

\section{ASCLEPIADACEAE}

Peripoloca angustifolia Labill. NPh., (O1, AS1-2-3, $\mathrm{SS}, \mathrm{SC}$ et $\mathrm{SO}), \mathrm{AC}$.

\section{ASTERACEAE}

Anacyclus clavatus (Desf.) Pers. Th., (O1-2-3, A12, K1-2-3, C1, H1-2, Hd et AS1-2-3), C.

Andryala integrifolia L. H., (O1-2-3, A1-2, K1-2-3, 
C1, H1-2, Hd et AS1-2-3), C.

Artemisia herba-alba Asso. Ch., (O1-2-3, C1, H, SS et SC), AC.

Asteriscus aquaticus (L.) Less. Th., (O1-2-3, A1-2, K1-2-3 et C1), AC.

Atractylis cancellata L. Th., (O1-2-3, A1-2, K1-2-3, C1, H1-2, Hd et AS1-2-3), C.

Bellis sylvestris Cirillo H., (O1-2-3, A1-2, K1-2-3 et $\mathrm{C} 1$ ), $\mathrm{C}$.

Bombycilaena discolor (Pers.) M. Lainz Th., (O1-23, A1-2, K1-2-3, C1, H1-2, Hd et AS1-2-3), C.

Calendula arvensis $\mathrm{L}$. Th., (O1-2-3, A1-2, K1-2-3, C1, H1-2, Hd et AS1-2-3), C.

Carlina lanata $\mathrm{L}$. Th., (O1-2-3, A1-2, K1-2-3, C1, H1-2, Hd et AS1-2-3), C.

Carthamus lanatus L. Th., (O1-2-3, A1-2, K1-2-3 et $\mathrm{C} 1$ ), $\mathrm{C}$.

Catananche caerulea L. H., (O1-2-3, A1-2, K1-2-3 et $\mathrm{C} 1$ ), $\mathrm{C}$.

Centaurea africana Lam. H., (O1-2-3, A1-2, K12-3 et $\mathrm{C} 1$ ), AC.

Centaurea amara subsp. angustifolia Gremli H., (O1), R.

Centaurea aspera L. H., (localité non disponible), $\mathrm{R}$.

Centaurea calcitrapa L. H. (O1-2-3, A1-2, K1-2-3, C1, H1-2, Hd et AS1-2-3), C.

Centaurea eriophora L. Th., (O1-2-3, A2, K1-2 et H1-2), [AMI], R.

Centaurea fragilis Durieu Ch., (O1-2-3), [AM], C.

Centaurea involucrata Desf. Th., (A1-2 et H1-2), [AM], AC.

Centaurea melitensis L. H.(Th.), (O1-2-3, A1-2, K1-2-3, C1, H1-2, Hd et AS1-2-3) AC.

Centaurea pullata L. Th.(H.), (O1-2-3, A1-2, K12-3 et C1), AC.

Cichorium intybus L. H., (O1-2-3, A1-2, K1-2-3, C1, H1-2, Hd et AS1-2-3), C.

Chrysanthemum coronarium L. Th., (O1-2-3, A12, K1-2-3 et C1), C.

Cladanthus arabicus (L.) Cass. Th., (O1-2), AC.

Conyza canadensis (L.) Cronquist Th., (O1-2-3, A1-2, K1-2-3, C1, H1-2, Hd et AS1-2-3), C.

Crepis amplexifolia (Godr.) Willk. Th., (O1-2-3, A1-2 et C1), [Afn], C.

Crepis salzamanii Bab. H., (O1), [AM], R.

Crepis vesicaria subsp. hanseleri (Boiss.) P.D. Sell H., (O1-2-3, A1-2 et K1-2-3), C.
Dittrichia viscosa (L.) Greuter Ch., (O1-2-3, A1-2, $\mathrm{K} 1-2-3$ et C1) C.

Echinops strigosus L. Th., (O1-2-3), [AfnI], C.

Eupatorium cannabinum L. H.(G.), (O3, K1-2 ), R.

Filago duriaei Coss. ex Lange Th., (O3 et H1), [AMI], R.

Filago congesta Guss. ex DC. Th., (O2-3 et H1-2), [AM], R.

Filago heterantha subsp. dichotoma (Pomel) Murb. Th., (O3), R.

Filago pyramidata $\mathrm{L}$. Th., (O1-2-3, A1-2, K1-2-3, C1, H1-2, Hd et AS1-2-3), C.

Galactites duriaei Spach ex Durieu H., (O1), [AMI], C.

Galactites tomentosa Moench H., (O1-2-3, A1-2, K1-2-3 et C1), C.

Glossopappus macrotus (Durieu) Briq. Th., (O12-3), [AfnI], C.

Hedypnois rhagadioloides (L.) F.W. Schmidt Th., (O1-2-3, A1-2, K1-2-3 et C1), C.

Helichrysum stoechas (L.) Moench Ch., (O1-2-3, A1-2, K1-2-3 et C1), C.

Helminthotheca aculeata (Vahl) Lack H., (O1-2-3, A1-2, K1-2-3, C1 et AS1-2-3), AC.

Hyoseris radiata L. H., (O1-2-3, A1-2, K1-2-3, C1, H1-2, Hd et AS1-2-3), C.

Hyoseris scabra L. Th. (O1-2-3, A1-2, K1-2-3, C1), C.

Hypocheris achyrophorus L. Th. (O1-2-3, A1-2, K1-2-3, C1, H1-2, Hd et AS1-2-3), C.

Hypochoeris radicata L. H., (O1-2-3, A1-2, K1-2-3, C1, H1-2, Hd et AS1-2-3), C.

Inula crithmoides $\mathrm{L}$. Ch., (O1-2-3, A1-2, K1-2-3, C1, H1-2, Hd et AS1-2-3), AC.

Launaea arborescens (Batt.) Murb. Ch., (O1, AS1, $\mathrm{SS} 1$ et SO), AC.

Leontodon hispidulus (Delile) Boiss. H., (O1-23, A1-2, K1-2-3, C1, H1-2, Hd et AS1-2-3), [AfnI], C.

Leontodon longirostris (Finch \& P.D.Sell) Talavera Th., (O1-2-3, A1-2, K1-2-3, C1, H1-2, Hd et AS1-2-3), C.

Leontodon tuberosus L. G., (O1-2-3, A1-2, K1-2-3, C1, H1-2, Hd et AS1-2-3), C.

Logfia gallica (L.) Coss. \& Germain Th., (O1-2-3, A1-2, K1-2-3, C1, H1-2, Hd et AS1-2-3), C.

Mauranthemum paludosum (Poir.) Vogt et Oberprieler Th., (O1-2-3, A1-2, K1-2-3 et C1), 
[AfnI] C.

Pallenis maritima (L.) Greuter Ch., (O1-2-3, A1-2, K1-2-3 et C1), C.

Pallenis spinosa (L.) Cass. H., (O1-2-3, A1-2, K12-3, C1, H1-2, Hd et AS1-2-3) C.

Phagnalon saxatile (L.) Cass. Ch., (O1-2-3, A1-2, K1-2-3, C1, H1-2, Hd et AS1-2-3), C.

Picnomon acarna (L.) Cass. Th., (O1-2-3, A1-2, $\mathrm{K} 1-2-3$ et $\mathrm{C} 1), \mathrm{AC}$.

Picris coronopifolia (Desf.) DC. Th., (SS, AS et H1-2), [Afn.], R.

Picris cupuligera (Durieu) Walp. Th., (O1-2 et C1), [Afn.], AC.

Pseudognalalium luteo-album (L.) Hilliard \& Burtt Th., (O1-2-3, A1-2, K1-2-3, C1, H1-2, Hd, AS12-3, SS1-2, SC et SM), AC.

Pulicaria dysenterica (L.) Bernh. H., (O1-2-3, A1-2, K1-2-3, C1, H1-2, Hd et AS1-2-3), AC.

Pulicaria odora (L.) Rchb. H., (O1-2-3, A1-2, K12-3, C1, H1-2, Hd et AS1-2-3) C.

Reichardia intermedia (Sch. \& Bip.) Samp. H.(Th.), (O1-2-3, A1-2, K1-2-3, C1, H1-2, Hd et AS12-3), C.

Reichardia tingitana (L.) Roth Th.(H.), (O1), C.

Rhagadiolus stellatus (L.) Gaerth. Th., (O1-2-3, A1-2, K1-2-3, C1, H1-2, Hd et AS1-2-3), AC.

Scolymus maculatus L. Th., (O1-2-3, A1-2, K12-3, C1), AC.

Scolymus hispanicus L. H., (O1-2-3, A1-2, K1-2-3, C1, H1-2, Hd et AS1-2-3), AC.

Scorzonera laciniata L. H., (O1-2-3, A1-2, K1-2$3, \mathrm{C} 1), \mathrm{C}$.

Senecio leucanthemifolius Poir. Th., (O1-2-3, A1-2, $\mathrm{K} 1-2-3, \mathrm{C} 1) \mathrm{C}$.

Senecio lividus subsp. foenicuaceus (Ten.) Br. Bl. et Maire Th., (O1-2-3, A1-2, K1-2-3, C1) AC.

Senecio vulgaris $\mathrm{L}$. Th. (O1-2-3, A1-2, K1-2-3, C1, H1-2, Hd et AS1-2-3)C.

Sonchus asper (L.) Hill. Th.(H.) (O1-2-3, A1-2, $\mathrm{K} 1-2-3, \mathrm{C} 1, \mathrm{H} 1-2$, Hd et AS1-2-3), C.

Sonchus oleraceus L. Th. (O1-2-3, A1-2, K1-2-3, C1, H1-2, Hd et AS1-2-3), C.

Sonchus tenerrimus L. Th.(H.), (O1-2-3, A1-2, $\mathrm{K} 1-2-3, \mathrm{C} 1), \mathrm{C}$.

Taraxacum obovatum subsp. ochrocarpum Soest H., (O1-2-3, A1-2, K1-2-3, C1), C.

Tolpis umbellata Bertoi. Th., (O1-2-3, A1-2, K1-23, C1, H1-2, Hd et AS1-2-3), AC.

Urospermum picroides (L.) Scop. ex F.W. Schmidt Th., (O1-2-3, A1-2, K1-2-3, C1), C.

\section{BORAGINACEAE}

Borago officinalis L. Th. [Méd. SW. Asie et Macaronésie] C.

Cynoglossum cheirifolium L. Th., (O1-2-3, A1-2, K1-2-3, C1, H1-2, Hd et AS1-2-3), C.

Cynoglossum clandestinum Desf. H., (O1-2-3, A12, K1-2-3, C1, H1-2, Hd et AS1-2-3), C.

Echium humile subsp. pycnanthum (Pomel) Greuter \& Burdet H., (O1-2-3), [AfnI], C.

Lithodora prostata subsp. lusitanica (Samp.) Valdés Ch., (O1), R.

Neatostema apulum (L.) I. M. Th., (O1-2-3, A1-2, K1-2-3, C1, H1-2, Hd et AS1-2-3), AC.

\section{BRASSICACEAE}

Alyssum simplex Rudolphi Th., (O1-2-3, A1-2, K1-2-3 et C1), C.

Arabis auriculata Lam. Th., (O1-2-3, A1-2, K1-2-3, C1, H1-2, Hd et AS1-2-3), AC.

Biscutella baetica boiss. \& Reut. Th., (O1-2-3, A1-2, K1-2-3, C1, H1-2, Hd, AS1-2-3 et SS12), [AMI] C.

Brassica souliei subsp. amplexicaulis (Desf.) Greuter \& Burdet Th., (O1-2-3, A1-2, K1-2-3, C1, H1-2, Hd et AS1-2-3), [AMI], C.

Brassica fruticulosa subsp. cossoniana (Boiss.\& Reuter) Maire H.(Th.), (H1-2), [AMI], C.

Brassica maurorum Durieu Th., (O1-2-3), [AM], R.

Brassica tournefortii Gouan Th., (O1-2-3, A1-2, H1-2, Hd et AS1-2-3), R.

Carrichtera annua (L.) DC. Th., (O1-2-3, A1-2, K1-2-3, C1, H1-2, Hd et AS1-2-3), C.

Cordylocarpus muricatus Desf. Th., (O1-2-3, H1-2 et SS), [AM], AC.

Diplotaxis virgata (Cav.) DC. Th., (O1-2-3, A1-2, K1-2-3, C1, H1-2, Hd et AS1-2-3), [AMI], AC.

Eruca vesicaria (L.) Cav. Th., (O1-2-3, A1-2, K12-3, C1, H1-2, Hd, AS1-2-3 et SS), AC.

Erucastrum varium Dur Th., (O1-2-3, A2, C1, H12, Hd et AS1-2-3), [Afn.], AC.

Hirshfeldia incana (L.) Lagr.-Foss. H., (O1-2-3, A1-2, K1-2-3, C1, H1-2, Hd et AS1-2-3), AC.

Lobularia maritima (L.) Desv. Ch., (O1-2-3, A1-2, K1-2-3, C1, H1-2, Hd et AS1-2-3), C.

Matthiola fruticulosa (Loefl. ex L.)Maire Ch., (O1-2-3, A1-2, K1-2-3, C1, H1-2, Hd et AS12-3), C. 
Matthiola parviflora (Schousb.) R. Br. Th. (O1-2-3, A1-2, K1-2-3, C1, H1-2, Hd et AS1-2-3), AC.

Matthiola tricuspidata (L.) R. Br. Th. (O1-2-3, A1 et K1-2-3,), AC.

Raphanus rhaphanistrum L. Th., (O1-2-3, A1-2, K1-2-3, C1, H1-2, Hd et AS1-2-3), AC.

Sinapis alba subsp. dissecta (Lag.) Bonnier Th., (O1-2-3, A1-2, K1-2-3 et C1), AC.

Sinapis arvensis $\mathrm{L}$. Th. (O1-2-3, A1-2, K1-2-3, C1, H1-2, Hd et AS1-2-3), AC.

Teesdalia coronopifolia (J. P. Bergeret) Thell. Th., (O1-2-3, A1-2, K1-2-3, C1, H1-2, Hd et AS12-3), AC.

\section{CACTACEAE}

Opuntia ficus indica auct. NPh., (localité non disponible), C.

\section{CAMPANULACEAE}

Campanula kremeri Boiss. \& Reuter Th., (O1-3, A1, K1-2-3, et AS3), [Afn.], AC.

Campanula trachelium subsp. mauritanica (Pomel) Quèzel H. (O1-2-3, A1-2, K1-2-3, C1, Hd et AS3), AC.

Legousia falcata (Ten.) Janch. Th., (O1-2-3, A1-2, $\mathrm{K} 1-2-3, \mathrm{C} 1), \mathrm{AC}$.

Trachelium coeruleum L. Ch., (O1-2-3, A1-2, K12-3 et $\mathrm{C} 1$ ), C.

Wahlenbergia lobelioides subsp. nutabunda (Guss.) Murb. Th., (O1), R.

\section{CAPRIFOLIACEAE}

Lonicera biflora Desf. Phl., (O1-2-3), [AMI], R.

Lonicera implexa L. Phl., (O1-2-3, A1-2, K1-2-3 et $\mathrm{C} 1$ ), AC.

Viburnum tinus L. Ph., (O1-2-3, A1-2, K1-2-3 et C1), AC.

\section{CARYOPHYLLACEAE}

Arenaria emarginata subsp emarginata Th., (O1-3 et A1), [AMI], R.

Arenaria leptoclados (Reichenb.) Guss. Th., (A2), $\mathrm{R}$.

Arenaria serpyllifolia L. Th., (O1-2-3, A1-2, K12-3, C1 et AS1-2-3), AC.

Cerastium brachypetalum Pers. Th., (O1-2-3, A1-2,
K1-2-3,C1 et AS1-2-3), AC.

Dianthus sylvestris subsp. siculus (Presl) Tutin H., (O1 et A1) AC.

Herniaria hemistemon J. Gay Ch.(H., Th.), (H1-2, AS3 et SS), AC.

Paronychia arabica (L.) DC. Th., (AS, SS), C.

Paronychia echinulata A.O. Chater Th. (O1-2-3, A1-2, K1-2-3 et C1), C.

Polycarpon tetraphyllum (L.) L. Th.(Ch.), (O1-2-3, A1-2, K1-2-3, C1, H1-2 et Hd), AC.

Pteranthus dichotomus Forssk. Th., (O2-3, H1-2, SS et SC), AC.

Silene behen $\mathrm{L}$. Th., (O1 et A1), C.

Silene colorata subsp.trichocalycina Fenzl Th., (O1-2-3), C.

Silene gallica L. Th., (O1-2-3, A1-2, K1-2-3, C1 et AS3), AC.

Silene nicaeensis All. Th., (O1-2-3, A1-2, K1-2-3, et AS1), AC.

Silene nocturna L. Th., (O1-2-3, A1-2, K1-2-3, C1 et AS1-2-3), AC.

Silene tridentata Desf. Th. (O1-2-3, K1, A1, H1 et AS1), R.

Silene vulgaris (Moench) Garcke Ch. (H.), (O1-2-3, A1-2, K1-2-3, C1 et AS1-2-3), C.

Spergularia diandra (Guss.) Boiss. Th., (O1-2-3, A1-2, K1-2-3, C1, H1-2, Hd, AS1-2-3 et SS), C.

Vaccaria hispanica (Mill.) Rauschert Th., (O1-2-3, A1-2, K1-2-3, C1, H1-2, Hd, AS1-2-3, SS1-2, $\mathrm{SC}$ et $\mathrm{SM}), \mathrm{C}$.

\section{CHENOPODIACEAE}

Arthrocnemum macrostachyum (Moric.) Moris NPh. (O1-2-3, A1-2, K1-2-3, C1, H1-2, Hd, AS1-2-3 et SS1-2), R.

Chenopodium murale L. Th., (O1-2-3, A1-2, K1-2-3, C1, H1-2, Hd, AS1-2-3, SS1-2, SC et $\mathrm{SM}), \mathrm{C}$.

Salsola kali L. Th., ((O1-2, A1, K1-2-3, et H1), C. Sarcocornia perennis (Mill.) A.J.Scott NPh., (O1), R.

Suaeda vera J. F. Gmelin NPh. (O1-2-3, A1-2, K12-3, C1, H1-2, Hd, AS1-2-3, SS1-2), AC.

\section{CISTACEAE}

Cistus albidus L. NPh., (O3, A1-2, C1 et H1-2), C. Cistus clusii Dunal NPh., (O1-3, A2, H1 et AS12), C. 
Cistus creticus L. Ch.(NPh.), (O1-3, A1-2 et AS1), AC.

Cistus heterophyllus Desf. NPh., (O1 et A1), [AM], AC.

Cistus ladaniferus L. NPh., (O1-3 et A1-2), C.

Cistus monspeliensis L. NPh. (O1-2-3, A1-2, K12-3, C1, H1-2, Hd, AS1-2-3), C.

Cistus munbyi Pomel NPh., (O1-3 et A1), [AM.], R.

Cistus salviifolius L. Ch.(NPh.), (O1-2-3, A1-2, K1-2-3 et C1), AC.

Fumana laevipes (L.) Spaech Ch., (O1-2-3, A1-2, K1-2-3, C1, H1-2, Hd et AS1-2-3) C.

Fumana thymifolia (L.) Spach ex Webb Ch. (O1-23, A1-2, K1-2-3, C1, H1-2, Hd et AS1-2-3), C.

Halimium halimifolium (L.) Willk. Ch., (O1-3, K1-2-3 et H1), AC.

Halimium umbellatum subsp. viscosum Willk. Ch., (O3), [AMI], R.

Helianthemum apenninum (L.) Mill. Ch., (O1-3), R.

Helianthemum helianthemoides (Desf.) Grosser Ch., (O3, K1-2 et H1), [Afn], C.

Helianthemum marifolium subsp. molle (Cav.) G.López Ch., (O1), [AMI] R.

Helianthemum pomeridianum Dunal Ch., (O1-2), [AM], C.

Helianthemum syriacum (Jacq.) Dum. Cours. Ch., (O1-2-3, A1-2, K1-2-3 et C1), C.

Helianthemum viscarum Boiss. \& Reut. Ch., (O12-3), [AMI], AC.

Tuberaria guttata (L.) Fourr. Th. (O1-2-3, A1-2, $\mathrm{K} 1-2-3$ et $\mathrm{C} 1), \mathrm{AC}$.

\section{CONVOLVULACEAE}

Convolvulus althaeoides L. H. (O1-2-3, A1-2, K12-3, C1, H1-2, Hd et AS1-2-3), C.

Convolvulus arvensis L. G. (O1-2-3, A1-2, K1-2-3, C1, H1-2, Hd et AS1-2-3), AC.

Convolvulus siculus subsp. elongatus Batt. Th., (O1-3), R.

Convolvulus tricolor $\mathrm{L}$. Th., (O1-2-3, A1-2, K1-2-3 et $\mathrm{C} 1$ ), AC.

Convolvulus valentinus subsp. suffruticosus (Desf.) Maire Ch., (O1-3), [AMI] AC.

Cuscuta approximata Bab. Th., (O1-2-3, A1-2, K1-2-3, C1 et AS3), AC.

\section{CRASSULACEAE}

Sedum amplexicaule subsp. tenuifolium ( $\mathrm{Sm}$.) Greuter Ch., (O1-2-3, A1-2, K1-2-3, C1 et
AS3), R.

Sedum maireanum Sennen Ch., (O1), [AM], R.

Sedum sediforme (Jacq.) Pau Ch. (O1-2-3 et K12-3), C.

Umbilicus rupestris (Salisb.) Dandy H., (O1-2-3, A1-2, K1-2-3 et C1), C.

\section{DIPSACACEAE}

Scabiosa atropurpurea L. Th.(H.), (O1-2-3, A1-2, K1-2-3, C1, H1-2, Hd et AS1-2-3), C.

Scabiosa semipapposa DC. Th.(H.), (O1-2-3, A1-2, K1-2-3, C1, H1-2, Hd et AS1-2-3), [AMI], C.

Scabiosa stella L. Th., (O1-2-3, A1-2, K1-2-3, C1, H1-2, Hd et AS1-2-3), C.

\section{ERICACEAE}

Arbutus unedo L. NPh., (O1-2-3, A1-2, K1-2-3, $\mathrm{C} 1$ et AS1-2), C.

Erica arborea L. NPh., (O1-2-3, A1-2, K1-2-3, C1, AS1-3), C.

Erica multiflora L. NPh. (O1-2-3, A1-2 et K1-2-3), C.

\section{EUPHORBIACEAE}

Euphorbia exigua L. Th. (O1-2-3, A1-2, K1-2-3, C1, H1-2, Hd et AS1-2-3), C.

Euphorbia falcata L. Th. (O1-2-3, A1-2, K1-2-3, C1, H1-2 et Hd) AC.

Euphorbia peplus L. Th. (O1-2-3, A1-2, K1-2-3, C1, H1-2, Hd, AS1-2-3, SS1-2, SC et SM), C.

Euphorbia pterococca Brot. Th., (O1-2-3, A1-2, K1-2-3 et C1), AC.

Euphorbia serrata L. Th., (O1 et A2), AC.

Euphorbia sulcata Lens ex Loisel Th., (O1-2-3, H1-2, AS1-2-3), AC.

Euphorbia terracina L. Th., (O1-2-3, A1-2, K1-2-3, C1, H1-2, Hd et AS1-2-3) C.

\section{FAGACEAE}

Quercus coccifera $\mathrm{L}$. NPh.(Ph), (O1-2-3, A1-2, K1-2-3, C1et AS3), C.

Quercus rotundifolia Lam. Ph., (O1-2-3, A1-2, K1-2-3, C1), AC.

Quercus suber L. Ph. (O1-3, A1-2, K1-2-3 et C1), AC.

\section{FRANKENIACEAE}

Frankenia composita Pau \& Font Quer Ch., (O1), R. 


\section{FUMARIACEAE}

Fumaria capreolatea $\mathrm{L}$. Th. (O1-2-3, A1-2, K1-2-3, C1, H1-2, Hd et AS1-2-3), C.

Fumaria densiflora DC. Th. (O1-2-3, A1-2, K1-2-3 et AS1-2-3) C.

\section{GENTIANACEAE}

Blackstonia perfoliata subsp. perfoliata Maire Th., (K2-3), R.

Blackstonia perfoliata subsp. grandiflora(Viv.) M. Th., ((O1-2-3, A1-2, K1-2-3, C1, H1-2 Hd et AS1-2-3), C.

Centaurium erythraea Rafin. H.(Th.), (O1-2-3, A1-2, K1-2-3 et C1), C.

Centaurium pulchellum (Sw.) Druce Th. (O1-2-3, A1-2, K1-2-3, C1, H1-2, Hd et AS1-2-3), C.

Exaculum pusillum (Lam.) Caruel Th. (A1 et K1-2-3), R.

\section{GERANIACEAE}

Erodium aethiopicum (Lam.) Brumh. \& Thell. Th., (O1-3, A2, K1-2-3 et C1), C.

Erodium chium (L.) Willd. Th., (O1-2-3, A1-2, $\mathrm{K} 1-2-3$ et $\mathrm{C} 1$ ), C.

Erodium cicutarium (L.)L'Hér. Th., (O1-2-3, A12, K1-2-3, C1, H1-2, Hd, AS1-2-3, SS1-2, SC et $\mathrm{SM}), \mathrm{C}$.

Erodium laciniatum (Cav.) Willd. Th., (O1-2 et A1), AC.

Erodium malachoides subsp. malacoides Maire Th., (O1-2-3, A1-2, K1-2-3, C1, H1-2, Hd, et AS1-2-3), C.

Erodium neuradifolium Delile Th., (O1, A1, et $\mathrm{SC}), \mathrm{AC}$.

Geranium molle L. Th., (O1-2-3, A1-2, K1-2-3, C1, H1-2, Hd et AS1-2-3), C.

Geranium purpureum Vill. Th.(H.), (O1-2-3, A1-2, $\mathrm{K} 1-2-3, \mathrm{C} 1, \mathrm{H} 1-2$, Hd et AS1-2-3)C.

Geranium rotundifolium $\mathrm{L}$. Th. (O1-3, A1-2, K12-3 et C1) AC.

\section{GLOBULARIACEAE}

Globularia alypum L. Ch. (O1-2-3, A1-2, K1-2-3, C1, H1-2, Hd et AS1-2-3), C.

\section{GUITTIFERAE}

Hypericum perfoliatum L. H., (O1-2-3, A1-2, K1-
2-3 et $\mathrm{C} 1$ ), C.

Hypericum tomentosum $\mathrm{L}$. H., (O1-2-3, A1-2, K12-3, C1, H1-2, Hd et AS1-2-3), R.

\section{LAMIACEAE}

Ajuga chamaepytis (L.) Schreb. Th., (O1-2-3, A1-2, K1-2-3, C1, H1-2, Hd et AS1-2-3), C.

Ajuga iva (L.) Schreb. H.(Th.), (O1-2-3, A1-2, K1-2-3 et C1), C.

Ajuga reptans L. H., (K1-2), R.

Lamium amplexicaule L. Th., (O1-2-3, A1-2, K12-3, C1, H1-2, Hd et AS1-2-3), C.

Lavandula dentata L. Ch., (O1-2 et A1), [AMI] C.

Lavandula multifida L. Ch., (O1-2-3 et K1-2-3) [Afn.] AC.

Lavandula stoechas $\mathrm{L}$. Ch., (O1-2-3, A1-2, K1-2-3 et C1), C.

Marrubium vulgare L. Ch., (O1-2-3, A1-2, K1-2-3, $\mathrm{C} 1, \mathrm{H} 1-2, \mathrm{Hd}$ et AS1-2-3), C.

Phlomis herba venti $\mathrm{L}$. H., (O1-2-3, A1-2, K1-2-3, C1, H1-2, Hd et AS1-2-3), R.

Prasium majus L. NPh., (O1-2-3, A1-2, K1-2-3, C1, H1-2, Hd et AS1-2-3), C.

Rosmarinus officinalis L. NPh., (O1-2-3, A1-2, K1-2-3, C1, H1-2, Hd et AS1-2-3) C.

Rosmarinus eriocalyx Jord. \&Fourr. NPh., (O1-2-3, A1-2 et H1), [AfnI], C.

Salvia verbenaca L. H., (O1-2-3, A1-2, K1-2-3, C1, H1-2, Hd et AS1-2-3), C.

Satureja barceloi (Willk.) Pau Ch., (O1-3 et A1), [AfnI], C.

Satureja graeca L. Ch., (O1-2-3, A1-2, K1-2-3, C1, H1-2, Hd et AS1-2-3), AC.

Satureja nepta (L.) Sheele Ch. (O1-2-3, A1-2, K12-3 et $\mathrm{C} 1), \mathrm{C}$.

Stachys ocymastrum (L.) Briq. Th. (O1-2-3, A1-2, K1-2-3, C1, H1-2, Hd et AS1-2-3), C.

Teucrium capitatum L. Ch. (O1-2-3, A1-2, K1-2-3, C1, H1-2, Hd et AS1-2-3)C.

Teucrium maghrebinum Greuter \& Burdet Th., (O1), [AM] R.

Teucrium fruticans $\mathrm{L}$. Ch. (O1-3 et H1) AC.

Teucrium pseudo-champaepytis L. Ch., (O1-2-3, A1-2, K1-2-3 et C1), C.

Teucrium pseudo-scorodonia Desf. Ch., (O1, A2, K1-2-3 et C1), [AfnI] C.

Teucrium resupinatum Desf Th., ((O1-2-3, A1-2, K1-2-3 et C1),[AMI] AC.

Thymbra captitata (L.) Cav. Ch., (O3), AC. 
Thymus munbyanus subsp. coloratus (Boiss. \& Reut.) Greuter \& Burdet Ch., (O1-2-3, A1-2, $\mathrm{K} 1-2-3$ et $\mathrm{C} 1$ ) [AM] AC.

Thymus munbyanus subsp. munbyanus Ch. (O12- 3 et H1-2), [AM], AC.

\section{LEGUMINOSAE}

Amphinomia lupinifolia (Boiss.) Pau Ch. (H.), (O1), [AMI], R.

Anagyris foetida $\mathrm{L}$. NPh., (O1-2-3, A1-2, K1-2-3 et $\mathrm{C} 1$ ), C.

Anthyllis vulneraria L. Th.(H.), (O1-2-3, A1-2, K1-2-3 et C1), C.

Astragalus echinatus Murray Th., (O1-2-3, A1-2, K1-2-3 et C1), C.

Astragalus epiglottis L. Th., (O1-2-3, A1-2, K1-2-3 et $\mathrm{C} 1$ ), $\mathrm{C}$.

Astragalus hamosus L. Th., (O1-2-3, A1-2, K1-2-3, C1, H1-2, Hd, AS1-2-3 et SS2) AC.

Astragalus longidentatus Chater Th., (O1-3), [AMI], R.

Astragalus pelecinus (L.) Barneby Th., (O1-2-3, A1-2, K1-2-3 et C1), AC.

Astragalus scorpioides Pourr. Th., (O1-2-3 et H12), [AMI], AC.

Astragalus sesameus L. Th., (O1-2-3, A1-2, K1-2-3, C1, H1-2, Hd et AS1-2-3), C.

Bituminaria bituminosa (L.) Stirton in Bothalia H., (O1-2-3, A1-2, K1-2-3, C1, H1-2, Hd et AS1-2-3), C.

Calicotome intermedia C. Presl. NPh, (O1-2-3), C.

Calicotome villosa (Poiret) Link NPh., (K2-3), AC.

Ceratonia siliqua L. Ph. (O1-2-3, A1-2, K1-2-3, $\mathrm{C} 1$ et AS1-2-3) C.

Colutea atlantica Browicz NPh., (O3, A2 et AS23), [AMI], C.

Coronilla scorpioides (L.) W.D.J. Koch Th., (O1-23, A1-2, K1-2-3, C1, H1-2, Hd et AS1-2-3), C.

Coronilla valentina subsp. glauca (L.) Batt. NPh., (O1-2-3, A1-2, K1-2 et AS3), AC.

Coronilla valentina subsp. pentaphylla (Desf.) Batt. NPh., (O1-2-3, A1-2, K1-2-3 et C1), AC.

Cytisus villosus Pourr. NPh., (O1-3, A1-2, C1), R.

Ebenus pinnata Aiton Ch., (O1-2-3, A1-2, K1, H12, Hd, AS1-2-3), [AFN], C.

Genista caballeroi Pau. NPh., (O1), [AM], C.

Genista cephalantha subsp. cephalantha Maire
NPh., (O1), [AM], AC.

Genista hirsuta subsp. erioclada (Spach.) Raynaud NPh., (O1-2-3), [AM], C.

Genista quadriflora Munby NPh., (O1-2-3), [AM.], $\mathrm{R}$.

Genista tricuspidata Desf. NPh., (O1-2-3, A1-2, K1-2-3,C1 et SS1) [Afn.] C.

Hammatolobium Kremerianum (Coss.) C. Muell. Ch., (O1), [AM], R.

Hedysarum aculeolatum Boiss. Th., (O1), [AM] AC.

Hedysarum boveanum Bunge ex Basiner Ch., (O1-2-3, A2, $\mathrm{C} 1$ et $\mathrm{H} 1)$, R.

Hedysarum pallidum Desf. H., (O1-2-3, C1 et H1), [Afn.], C.

Hedysarum saxatile L. Ch., (Localité non disponible), R.

Hedysarum spinosissimum subsp. capitatum (Desf.) Asch. \& Gr. Th., (O1-2-3, A1-2, K1-2-3, $\mathrm{C} 1$ et H2), C.

Hippocrepis ciliata Willd. Th., (O1-2-3, A1-2, K12-3, C1, H1-2, Hd et AS1-2-3), C.

Hippocrepis multisilliquosa L. Th., (O1-2-3, A1-2, K1-2-3, C1, AS1-2-3 et SS1-2) C.

Lathyrus clymenum L. Th., (K2-3), C.

Lathyrus tingitanus $\mathrm{L}$. Th., (O1 et A1), R.

Lotus corniulatus L. H., (O1-2-3, A1-2, K1-2-3, $\mathrm{C} 1$ et AS3), AC.

Lotus cystisoides L. H., (O1-2-3, A1-2, K1-2-3 et $\mathrm{C} 1$ ), C.

Lotus creticus L. Ch., (O1-2, A1 et K1-2-3), C.

Lotus ornithopodioides L. Th., (O1-2-3, A1-2, K12-3 et $\mathrm{C} 1), \mathrm{C}$.

Lupinus hirsutus L. Th., (O1-2-3, A1-2, K1-2-3 et C1), AC.

Medicago doliata Carmign Th., (O1-2-3, A1-2, K12-3, C1, H1-2, Hd et AS1-2-3), AC.

Medicago litoralis Rhode ex Loisel Th., (O1-2-3, A1-2, C1, H1-2, AS1-2-3 et SS), C.

Medicago marina L. Ch., (O1-2, A1 et K1-2-3), AC.

Medicago minima L. Bartal. Th., (O1-2-3, A1-2, K1-2-3, C1, H1-2 et AS1-2-3), C.

Medicago polymorpha L. Th., (Localité non disponible), AC.

Medicago truncatula Gaertn. Th., (O1-2-3, A1-2, K1-2-3 et C1), C.

Melilotus speciosus Durieu Th., (O1-2), [AMI], R.

Onobrychis crista-galli (L.) Lam. Th., (O1-2-3), AC. 
Ononis euphrasiifolia Desf. Th., (O1-2-3), [AMI], $\mathrm{R}$.

Ononis laxiflora Desf. Th.(H.), (O1-2-3, A1-2, K12-3 et C1), [AfnI], AC.

Ononis mitissima L. Th., (O1-2-3, A1-2, K1-2-3 et C1), AC.

Ononis natrix L. Ch., (O1-2-3, A1-2, K1-2-3 et C1), C.

Ononis ornithopodioiodes L. Th., (O1-2-3, A2, C1, H1-2 et AS1-2-3), C.

Ononis pubescens L. Th., (O1-2-3, A1-2, K1-2-3, C1 et AS2), AC.

Ononis pusilla L. Ch., (O2-3, A2, C1, et AS1-23), AC.

Ononis reclinata subsp. mollis (Savi) Samp. Th., (O1-2-3, A1-2, K1-2-3, C1, H1-2, Hd et AS12-3), AC.

Ononis sicula Guss. Th., (O1-2-3, A1-2, K1-2-3, C1, H1-2, Hd et AS1-2-3), AC.

Ononis variegata $\mathrm{L}$. Th., (O1-2, A1-2, K1-2-3), AC.

Retama raetam subsp. bovei (Spach) Talavera et Gibbs. NPh., (O1 et K3), C.

Retama sphaerocarpa (L.) Boiss. NPh., (O3, C1, H1-2 et AS), R.

Scorpiurus sulcatus L. Th., (O1-2-3, A1-2, K1-2-3 et $\mathrm{C} 1$ ), C.

Scorpiurus vermiculatus $\mathrm{L}$. Th., (O1-2-3, A1-2, K1-2-3 et C1), C.

Trifolium angustifolium L. Th., (O1-2-3, A1-2, $\mathrm{K} 1-2-3, \mathrm{C} 1$ et AS3), C.

Trifolium arvense $\mathrm{L}$. Th., (O1-2-3, A1-2, K1-2-3, $\mathrm{C} 1$ et AS3), C.

Trifolium campestre Schreb. Th., (O1-2-3, A1-2, $\mathrm{K} 1-2-3, \mathrm{C} 1$ et AS1-2-3), C.

Trifolium cherleri L. Th., (O1-2-3, A1-2, K1-2-3, C1 et AS1-2-3), C.

Trifolium squarrosum $\mathrm{L}$. Th., (O1-2-3, A1-2, K12-3 et C1), C.

Trifolium stellatum $\mathrm{L}$. Th., (O1-2-3, A1-2, K1-2-3, $\mathrm{C} 1$ et AS3), C.

Trifolium tomentosum $\mathrm{L}$. Th.(H.), (O1-2-3, A1-2, K1-2-3 et C1), C.

Trigonella polyceratia L. Th., (O1-2-3, K1-2-3, H1-2, Hd, AS1-2-3, SS1-2 et SC), C.

Tripodion tetraphyllum (L.) Four. Th., (O1-2-3, A1-2, K1-2-3 et C1), C.

Ulex parviflorus subsp. africanus (Webb) Greuter NPh., (O1), [AM], AC.

Vicia hirsuta (L.) S.F. Gray Th., (O1-2-3, A1-2,
$\mathrm{K} 1-2-3$ et $\mathrm{C} 1)$, AC.

Vicia lathyroides $\mathrm{L}$. Th, (O1-2-3, A1-2, K1-2-3, C1 et AS1-2-3). AC.

Vicia lutea $\mathrm{L}$. Th., (O1-2-3, A1-2, K1-2-3 et C1), C.

Vicia onobrychoides L. H., (O1-2-3, A1-2, K1-2-3, $\mathrm{C} 1$, Hd et AS3), AC.

Vicia peregrina $\mathrm{L}$. Th., (O1-2, $\mathrm{A} 1-2$ et $\mathrm{C} 1), \mathrm{R}$.

Vicia pubescens (DC.) Link Th., (O1, A1 et K12-3), AC.

Vicia sativa L. Th. (O1-2-3, A1-2, K1-2-3 et C1), C.

\section{LINACEAE}

Linum munbyanum Boiss. \& Reut. H.(Ch.), (O12-3), [AM], R.

Linum strictum L. Th., (O1-2-3, A1-2, K1-2-3, C1, H1-2, Hd et AS1-2-3), AC.

Linum suffruticosum L. Ch., (O1-2-3, H1-2 et AS1-2-3), R.

Linum tenue Desf. Th., ((O1-2-3, A1-2, K1-2-3, C1 et $\mathrm{H} 1$ ), [AMI], C.

Linum trigynum $\mathrm{L}$. Th., (O1-2-3, A1-2, K1-2-3, C1, H1-2, Hd et AS1-2-3) R.

Linum usitatissimum L. Ch., (O1-2-3, A1-2, K1-23, C1, H1-2, Hd et AS1-2-3) AC.

\section{MALVACEAE}

Lavatera arborea $\mathrm{L}$. NPh., (O1 et A1), R.

Lavatera maritima Gouan NPh., (O1-2-3, A1-2, $\mathrm{K} 1-2-3$ et $\mathrm{C} 1$ ), $\mathrm{AC}$.

Lavatera olbia L. NPh., (O1-2-3, A1-2, K1-2-3 et C1) AC.

Malope malachoides subsp. malachoides $\mathrm{Ch}$. (Th.), (O1-2-3, A1-2, K1-2-3 et C1), R.

Malope malachoides subsp. stipularea (Cav.) Maire Ch. (Th.), (O1-2-3, A1-2, K1-2-3 et C1), [AMI], C.

Malope malachoides subsp. tripartita (Boiss. \& Reut.) Maire Ch. (Th.), (O2-3, C1 et H1-2), [AMI], AC.

Malva hispanica $\mathrm{L}$. Th .(H.), (O1-2-3 et A2), [IMA] C.

Malva sylvestris L. H., (O1-2-3, A1-2, K1-2-3, C1, H1-2, Hd, AS1-2-3, SS1-2), C.

\section{MORACEAE}

Ficus carica L. Ph., (O1-2-3, A1-2, K1-2-3 et $\mathrm{C} 1), \mathrm{C}$. 


\section{MYRTACEAE}

Myrtus communis L. Ph. (A1 et K1-2-3), C.

\section{OLEACEAE}

Jasmanium fruticans L. NPh., (O1-2-3, A1-2, K12-3, C1, AS1-2-3), AC.

Olea europea L. Ph. (O1-2-3, A1-2, K1-2-3, C1, AS1-2-3 et SS1-2), C.

Phillyrea angustifolia L. NPh., (O1-3, A2, K1-2-3, C1, AS1-2-3), C.

\section{OROBANCHACEAE}

Orobanche leptontha Pomel G., (O1), [AM], R.

Orobanche minor Sm. G., (O1-2-3, A1-2, K1-2-3, C1, H1-2, Hd, AS1-2-3 et SS1-2, SC), C.

Orobanche purpurea Jacq. G., (O2 et AS3), R.

Orobanche schultzii Mutel G., (O1-2-3, A1-2, K12-3 et $\mathrm{C} 1$ ), AC.

\section{OXALIDACEAE}

Oxalis pes-caprae L. G., (O1-2-3, A1-2, K1-2-3 et $\mathrm{C} 1$ ), $\mathrm{C}$.

\section{PAPAVERACEAE}

Glaucium flavum Crantz H., (O1-2, A1, K1-2-3), AC. Papaver rhoeas L. Th., (O1-2-3, A1-2, K1-2-3, C1, H1-2, Hd et AS1-2-3), C.

\section{PLANTAGINACEAE}

Plantago afra L. Th., (O1-2-3, A1-2, K1-2-3, C1, H1-2, Hd, AS1-2-3, SS1-2 et SC), C.

Plantago albicans L. H., (O1-2-3, A1-2, K1-2-3, C1, H1-2, Hd et AS1-2-3), C.

Plantago amplexicaule Cav. H., (O1-2-3, A1, H1-2, Hd, AS1-2-3, SS1-2 et SC) AC.

Plantago cupanii Guss. Th.(H.), (O1-2-3, A1-2, K1-2-3 et C1), AC.

Plantago lagopus L. Th., (O1-2-3, A1-2, K1-2-3, $\mathrm{C} 1, \mathrm{H} 1-2, \mathrm{Hd}$ et AS1-2-3), C.

\section{PLUMBAGINACEAE}

Limonium asparagoides (Coss. et Dur.) M. H., (O1), [AM], R.
Limonium delicatulum (Girard) Kuntze H., (O12-3), [AfnI], C.

Limonium echioides subsp. echioides Maire Th., (O1-2-3, A1-2, K1-2-3 et C1), C.

Limonium ferulaceum (L.) Chaz. Ch., (O2 et K3), R.

Limonium pescadense Greuter \& Burdet H., (O1-2, A1 et K1-2-3), C.

\section{POLYGALACEAE}

Polygala monspeliaca L. Th., (O1-2-3, A1-2, K12-3, C1, H1-2, Hd, AS1-2-3), C.

Polygala nicaensis Risso ex W.D.J. Koch H. (O1-23, A1-2, K1-2-3, C1, H1-2, Hd et AS1-2-3) C.

Polygala rupestris Pourr. Ch., (O1-2-3, A1-2, K12-3, C1 et AS1-2-3), AC.

\section{POLYGONACEAE}

Rumex bucephalophorus L. Th., (O1-2-3, A1-2, K1-2-3, C1 et AS1-2-3), C.

Rumex pulcher L. H. (O1-2-3, A1-2, K1-2-3 et C1), AC.

Rumex thyrsoides Desf. H., (O1-2-3, A1-2, K1-2-3, $\mathrm{C} 1$ et AS3), C.

\section{PRIMULACEAE}

Anagallis arvensis subsp. latifolia (L.) Arcang. Th., (O1-2-3, A1-2, K1-2-3 et C1), C.

Anagallis arvensis subsp. arvensis $\mathrm{L}$. Th., (O12-3, A1-2, K1-2-3, C1, H1-2, Hd, AS1-2-3 et SS1-2), C.

Asterolinon linum-stellatum (L.) Duby Th., (O1-23, A1-2, K1-2-3, C1, H1-2, Hd et AS1-2-3), C.

Coris monspeliensis L. Ch., (O1-2-3, A1-2, K1-2-3, C1, H1-2, Hd et AS1-2-3), AC.

\section{RAFLESIACEAE}

Cytinus hypocistis L. G., (O1-2-3, A1-2, K1-2-3 et $\mathrm{C} 1), \mathrm{C}$.

\section{RANUNCULACEAE}

Adonis aestivalis L. Th., (O1-2-3, A1-2, K1-2-3 et $\mathrm{C} 1$ ), AC.

Clematis cirrhosa L. Phl., (O1, A1 et K1-2-3), C.

Clematis flammula L. Phl., (O1, A1, K1-2-3, H1-2, 
AS1-2-3, SS1-2 et SC), AC.

Delphinium pentagynum Lam. H., (O1-3, A1-2, K1-2 et C1), [AMI], R.

Ranuncula paludosus Poir. H., (O1-2-3, A1-2, K12-3, C1, H1-2, Hd et AS1-2-3), C.

\section{RHAMNACEAE}

Rhamnus alteranus L. Ph., (O1-2-3, A1-2, K1-2-3, C1, H1-2, Hd et AS1-2-3), C.

Rhamnus lycioides subsp. oleoides (L.) Maire NPh., (O1-2-3, A1-2, K1-2-3, C1, H1-2, Hd, et AS1-2-3), AC.

Ziziphus lotus (L.) Lam. NPh., (O1-2-3, H1-2, Hd, AS1-2-3 et SS1-2), C.

\section{RESEDACEAE}

Reseda alba L. Th., (O1-2-3, A1-2, K1-2-3, C1, H1-2, Hd, AS1-2-3 et SS1-2), AC.

Reseda lutea subsp. neglecta (Müller Arg.) Ball. Th., (O1-2-3, A1-2, K1-2-3, C1, H1-2, Hd, AS12-3, SS1-2 et SC), AC.

Reseda luteola $\mathrm{L}$. Th., (O1-2-3, A1-2, K1-2-3 et C1), AC.

Reseda phyteuma subsp. collina (Müller Arg.) Ball. Th.(Ch.), (O1-2-3 et H1-2), [Afn.], C.

Reseda phyteuma subsp. phyteuma Th. (O1-2-3, A1-2, K1-2-3, C1, H1-2, Hd et AS1-2-3) R.

\section{ROSACEAE}

Crataegus monogyna Jacq. Ph., (O1-2-3, A1-2, K1-2-3, C1 et AS1-2-3), C.

Rosa canina L. Ph. (O1-2-3, A1-2, K1-2-3, C1 et AS3), C.

Sanguisorba minor subsp. alveolosa (Spach) Maire H., (O1-2-3), C.

\section{RUBIACEAE}

Asperula arvensis L. Th., (O1-2-3, A1-2, K1-2-3, C1, H1-2, Hd et AS1-2-3), R.

Asperula hirsuta Desf. Ch., (O1-2-3, A1-2, K1-2-3, C1, H1-2, Hd et AS1-2-3), [AfnI], C.

Crucianella maritima L. Ch., (O1-2, A1 et K12-3), C.

Galium spurium L. Th. (O1-2-3, A1-2, K1-2-3, C1, H1-2, Hd et AS1-2-3), AC.

Galium bourgeanum Coss. Ch., (O3), [AM.], AC.
Galium lucidum All. H., (O1-2-3, A1-2, K1-2-3, C1, H1-2, Hd et AS1-2-3), C.

Galium murale (L.) All. Th., (O1-2-3, A1-2, K1-23, C1, H1-2 et Hd, AS1-2-3), C.

Galium triconutum Dandy Th., (O1-2-3, A1-2, K12-3, C1, H1-2, Hd, AS1-2-3 et SS1-2), C.

Galium verticillatum Danth. Th., (O3, K1 H1-2, AS1-2-3), R.

Rubia peregrina L. Phl., (O1-2-3, A1-2, K1-2-3, $\mathrm{C} 1$ et AS1-2-3), C.

Sherardia arvensis L. Th., (O1-2-3, A1-2, K1-2-3, C1, H1-2, Hd et AS1-2-3), C.

Vaillantia hispida L. Th., (O1-2-3, A1-2, K1-2-3 et C1), AC.

\section{RUTACEAE}

Ruta angustifolia Pers. Ch., (O1-2-3, A1-2, K1-2-3, $\mathrm{C} 1, \mathrm{H} 1-2, \mathrm{Hd}$ et AS1-2-3), C.

\section{SALICACEAE}

Salix purperea L. Ph., (O1-2-3, A1-2, K1-2-3, C1 et AS1-2-3), AC.

\section{SANTALACEAE}

Osyris alba L. NPh., (O1-2-3, A1-2, K1-2-3, C1, AS1-2-3 et SC), AC.

Osyris quadripartita Salzm. NPh., ((O1-2-3 et A12), [AMI], AC.

Thesium humile Vahl. Th., (O1-2-3, A1-2, K1-2-3, C1, H1-2, Hd et AS1-2-3), AC.

\section{SAXIFRAGACEAE}

Saxifraga globulifera Desf. Ch., (O1-2-3, A1-2, $\mathrm{K} 1-2-3$ et $\mathrm{C} 1$ ), [AMI], C.

\section{SCROPHULARIACEAE}

Anarrhinum pedatum Desf. H., (O1-2-3, A1-2, K1-2-3 et C1), [Afn.], C.

Antirrhinum cirrhigerum (Welw. ex Ficalho) Rothm. Ch., (O1), [AM.], R.

Antirrhinum tortuosum Bosc ex Vent. Ch., (O13), R.

Bartsia trixago L. Th., (O1-2-3, A1-2, K1-2-3 et C1), C.

Kickxia commutata (bernh. ex Rchb.) Th.(H.), 
(O1-2-3, A1-2, K1-2-3 et C1), AC.

Linaria arvensis subsp. arvensis Th., (O1-3, A2, C1 et H1-2), C.

Linaria triphylla (L.) Mill. Th., (O1-2-3, A1-2, K12-3, C1, H1-2, Hd et AS1-2-3), C.

Misopates orontium (L.) Raf. Th., (O1-2-3, A1-2, K1-2-3, C1, H1-2, Hd, AS1-2-3 et SC), C.

Odontites purpurea subsp. purpurea Quézel et Santa Ch., (O1-2-3, A1-2, K1-2-3, C1, H1-2, Hd et AS1-2-3), [AMI], C.

Scrophularia canina L. Ch., (O1-2-3, A1-2, K1-2-3, C1, H1-2, Hd et AS1-2-3), C.

Veronica arvensis L. Th., (O1-2-3, A1-2, K1-2-3, C1, H1-2, Hd et AS1-2-3), C.

\section{SOLANACEAE}

Lycium intricatum Boiss. NPh., (O1-2), C.

Solanum sodomaeum L. NPh., (A1, K3 et C1), R.

Solanum nigrum L. Th., (O1-2-3, A1-2, K1-2-3, $\mathrm{C} 1, \mathrm{H} 1-2, \mathrm{Hd}, \mathrm{AS} 1-2-3$ et SC) C.

Withania frutescens (L.) Pauquy NPh., (O1-2), [AMI] C.

\section{THELIGONACEAE}

Theligonum cynocrambe L. Th., (O1-2-3, A1-2, $\mathrm{K} 1-2-3$ et $\mathrm{C} 1), \mathrm{AC}$.

\section{THYMELAECEAE}

Daphne gniduim L. NPh., (O1-2-3, A1-2, K1-2-3 et $\mathrm{C} 1$ ), C.

Thymelea argentata (Lam.) Pau Ch., (H1-2), [AfnI], R.

Thymelea hirusta (L.) Endl. NPh., (O1-2-3, A1-2, K1-2-3, C1, H1-2, Hd et AS1-2-3), AC.

\section{UMBELLIFERAE}

Ammoides pussilla (Bort.) Breistr. Th. (O1-2-3, A12, K1-2-3, C1, H1-2, Hd et AS1-2-3), C.

Bifora testiculata (L.) Roth. Th., (O1-2-3, A1-2, $\mathrm{K} 1-2-3$ et $\mathrm{C} 1), \mathrm{AC}$.

Bunium pachypodum P.W. Ball G. (localité non disponible), AC.

Bupleurum balansae Bois. \& Reut. Ch., (O1-2-3, A1-2, K1-2-3 et C1) [AM] AC.

Bupleurum gibraltaricum Lam. NPh., (O1-2-3), AC. Bupleurum semicompositum L. Th., (O1-2-3, A1-2,
K1-2-3, C1, H1-2, Hd, AS1-2-3), AC.

Crithmum maritimum L. Ch., (O1-3, A1 et K12-3), C.

Daucus carota subp. carota (L.) Thell. Th. (H.), (O1-2-3, A1-2, K1-2-3 et C1), C.

Daucus carota subsp. maximus (Lamk.) Sperg. Th.(H.), (O1-2-3, A1-2, K1-2-3 et C1), C.

Daucus carota subsp. parfilorus (Desf.) Fiori Th.(H.), (O1-2-3), [AM], R.

Daucus durieua Lange Th., (O3), AC.

Daucus muricatus (L.) L. Th. (O1-2-3, A1-2, K12-3, C1, H1-2, Hd et AS1-2-3), C.

Elaeoselinum fontanesii Boiss. H., (O1-2-3, A1-2, K1-2-3, C1, H1-2, Hd et AS1-2-3), [Afn], C.

Erygium campestre L. G., (O1-2-3, A1-2, K1-2-3, C1 et H1-2), R.

Erygium ilicifolium Lam. Th., (O1-2-3, H1-2, Hd, AS1-2-3 et SS1-2), [AfnI.], AC.

Erygium tricuspidata L. H.(G.), (O1-2-3, A1-2, K1-2-3, C1, H1-2, Hd et AS1-2-3), C.

Eryngium triquetrum Vahl. H., (O1-2-3, A1-2, K12-3, C1, H1-2, Hd et AS1-2-3), AC.

Ferula communis L., H. (O1-2-3, A1-2, K1-2-3, C1, H1-2, Hd, AS1-2-3, SS1-2 et SC), C.

Foeniulum vulgare Mill. H. (O1-2-3, A1-2, K1-2-3, C1, H1-2, Hd et AS1-2-3), C.

Kundmania sicula (L.) DC. H. (O1-2-3, A1-2, K12-3, C1, H1-2, Hd et AS1-2-3), C.

Pseudorlaya pumila (L.) Parl. Th., (O1-2, A1, K12-3, H1-2 et SS1-2), AC.

Scandix australis L. Th. (O1-2, A1, K1-2-3), C.

Scandix pecten-veneris $\mathrm{L}$. Th. (O1-2-3, A1-2, K12-3, C1, H1-2, Hd et AS1-2-3) C.

Thapsia transtagana Bort. H. (O1-2-3, A1-2 et H1-2), [AMI], C.

Torilis arvensis subsp. neglacta (Schult.) Thell. Th., (O1-2-3, A1-2, K1-2-3, C1, H1-2, Hd et AS1-2-3), C.

Trolis elongata ( Hoffm.\& Link) Samp. Th., (O1-23, A1-2, K1-2-3, C1, H1-2 et Hd), AC.

Trolis leptophylla (L.) Reichenb. Th. (O1-2-3, A1-2, K1-2-3, C1, H1-2, Hd et AS1-2-3), C.

Torilis nodosa (L.) Gaertn. Th. (O1-2-3, A1-2, K12-3 et C1), C.

\section{VALERIANACEAE}

Centranthus calcitrapae (L.) Dufr. H., (O1-2-3, A12, K1-2-3, C1, H1-2, Hd et AS1-2-3), C.

Centranthus macrosiphon Boiss. Ch., (O3), [AMI], AC. 
Fedia cornucopiae (L.) Gaertn. Th., (O1-2-3, A1-2, K1-2-3, C1, H1-2, Hd et AS1-2-3), [AMI] C.

Valerianella coronata (L.) DC. Th., (O1-3, A1-2, K1-2-3, C1 et AS1-2-3), C.

Valerianella muricata (M.Bieb.) J.W.Loudon Th., (O1-2-3, A1-2, K1-2-3, C1, H1-2, Hd, et AS12-3), AC.

\section{VIOLACEAE}

Viola arborescens L. Ch., (O1 et A1), C.

\section{ZYGOPHYLLACEAE}

Fagonia cretica L. Ch., (O1-2 et A1-2), C.

\section{MONOCOTYLEDONES ALLIACEAE}

Allium ampeloprasum L. G., (H1-2 et AS1-2-3), AC.

Allium neapolitanum Cirillo. G., (O1-2-3, A1-2, K1-2-3 et C1), C.

Allium nigrum L. G., (O1-2-3, A1-2, K1-2-3 et C1), C.

\section{AMARYLLIDACEAE}

Narcissus cantabricus DC. G., (O1-2-3 et AS2), [AMI], AC.

Narcissus elegans (Haw.) Baker G., (O1-2-3, A1-2, K1-2-3, C1 et H1-2), AC.

Narcissus tazetta L. G., (O1-2-3), C.

Pancratium foetidium var. foetidium G., (O1-3 et K2) [AM.] AC.

\section{ANTHERICACEAE}

Anthericum liliago subsp. algeriense (Boiss. \& Reut.) Maire \& Weiller G., (O1-2-3, A1-2, K12-3, C1 et AS1-2-3), [Afn.], C.

\section{APHYLLANTHACEAE}

Aphyllanthes monspeliensis L. H., (O1-2-3, A1 et $\mathrm{H} 1), \mathrm{C}$.

\section{ARACEAE}

Arisarum vulgare Targ. -Tozz. G., (O1-2-3, A1-2,
K1-2-3 et C1), C.

\section{ASPARAGACEAE}

Asparagus acutifolius L. Phl.(Ch.) (O1-2-3, A1-2, K1-2-3, C1 et AS1-2-3), C.

Asparagus albus L. Ch. (Phl.), (O1-2-3, A1-2, K12-3 et C1), AC.

Asparagus altissimus Munby Ch. (Phl.) (O1-3), [AM.], AC.

Asparagus horridus L. in Murray Ch. (Phl.), (O1-23, A1-2, K1-2-3, C1, H1-2, Hd et AS1-2-3), C.

\section{ASPHODELACEAE}

Asphodelus ayardii Jahand. \& Maire G. (O1-2-3, A1-2, K1-2-3, C1, H1-2, Hd et AS1-2-3), AC. Asphodelus ramosus L. G., (A1, H1-2 et AS1-23), C.

\section{COLCHICACEAE}

Colchicum lusitanicum Bort. G., (O1-2-3, A1-2, K1-2-3 et C1), AC.

Merendera filifolia Camb. G. (O1-2-3, A1-2, K12-3 et $\mathrm{C} 1$ ), AC.

\section{CYPERACEAE}

Carex hallerana Asso H., (O1-2-3, A1-2, K1-2-3, $\mathrm{C} 1$ et AS1-2-3), C.

\section{DISCOREACEAE}

Tamus communis L. G., (O1-2-3, A1-2, K1-2-3 et $\mathrm{C} 1), \mathrm{C}$.

\section{HYACYNTHACEAE}

Charybdis maritima (L.) Speta G., (O1-2-3, A1-2, K1-2-3 et C1), C.

Charybdis undulata (Desf.) Speta G., (O1-2-3, A12, K1-2-3 et C1), C.

Dipcadi serotinum (L.) Medik. G., (O1-2-3, H1-2, Hd et AS1-2-3)C.

Hyacinthoides lingulata (Poir.) Rothm. G., (O1-2-3, A1-2, K1-2-3 et C1), [Afn.], C.

Muscari comosum (L.) Mill. G., (O1-2-3, A1-2, K1-2-3, C1, H1-2, Hd et AS1-2-3), C.

Oncostema peruviana (L.) Speta G., (O1-2-3, A1-2, 
K1-2-3, C1, H1-2, Hd et AS1-2-3), C.

Ornithogalum sessiliflorum Desf. G., (O1-2-3, A1-

2, K1-2-3 et C1), [Afn.], AC.

Ornithogalum algeriense Jord. \& Fourr. G., (O12-3, A1-2, K1-2-3 et C1), [AfnI], C.

Prospero autumnale (L.) Speta G., (O1-2-3, A1-2, K1-2-3, C1 et AS1-2-3), C.

Prospero fallax (Steinh.) Speta G., (O1-2-3, A1-2, K1-2-3 et C1), [Afn.], AC.

\section{IRIDACEAE}

Gladiolus communis L. G., (O1-2-3, A1-2, K1-2-3, C1, H1-2, Hd et AS1-2-3), C.

Gladiolus segetum Ker-Gawl. G. (O1-2-3, A1-2, K1-2-3 et C1), C.

Gynandriris sisyrinchium (L.) Parl. G. (O1-2-3, A1-2, K1-2-3, C1, H1-2, Hd et AS1-2), C.

Iris tingitana Boiss. \& Reut. G., (O1-2-3 et A1-2), [AM], AC

Romulea bulbocodium (L.) Sebast. \& Mauri G. (O1-2-3, A1-2, K1-2-3, C1 et AS1-2-3) C.

\section{LILIACEAE}

Fritillaria lusitanica subsp. oranensis (Pomel) Valdés G., (O1 et K3), [Afn.] R.

Gagea algeriensis Chabert G., (O3 et A2), [AMI], $\mathrm{R}$.

Gagea durieui Parl. ex Trab. G., (O1-3), [AMI] C.

Gagea fibrosa (Desf.) Schult. \& Schult. G., (O3, H1-2 et AS1-2-3), AC.

\section{ORCHIDACEAE}

Anacamptis pyramidalis (L.) Rich. G. (O1-2-3, A1-2, K1-2-3, C1 et AS3), AC.

Gennaria diphylla (Link) Parl. G., (O1, A1, K2), AC.

Ophrys apifera Huds. G., (O1-2-3, A1-2, K1-2-3 et C1), AC.

Ophrys bombyliflora Link. G., (O1-2-3, A1-2, K12-3 et C1), R.

Ophrys dyris Maire G. (O1)[AMI] R.

Ophrys fusca Link. G. (O1-2-3, A1-2, K1-2-3 et C1), AC.

Ophrys lutea Cav. G., (O1-2-3, A1-2, K1-2-3, C1, H1-2, Hd, AS1-2-3), C.

Ophrys speculum Link. G., (O1-2-3, A1-2, K1-2-3 et $\mathrm{C} 1$ ), AC.
Ophrys sphegifera Willd. G., (O1-2-3, A1-2, K12-3, C1 et AS3), R.

Ophrys tenthredinifera Willd. G., (O1-2-3, A1-2, K1-2-3 et C1), C.

Orchis coriophora L. G. (O1-2-3, A1-2, K1-2-3 et C1), [AfnI], AC.

Orchis mascula (L.) L. G., (O1-2-3, A1-2, K1-2-3, $\mathrm{C} 1$ et AS3), AC.

Orchis champagneuxii Barnéoud G., (O1-3), AC.

Orchis papilionacea L. G., (O1-2-3, A1-2, K1-2-3, $\mathrm{C} 1$ et AS3), AC.

Serapias lingua L. G. (O1-2-3, A1-2, K1-2-3 et C1), AC.

Serapias parviflora Parl. G. (O1-2-3, A1-2, K1-2-3 et $\mathrm{C} 1$ ), $\mathrm{R}$.

PALMAE

Chamaerops humilis L. NPh. (Ch.), (O1-2-3, A1-2, K1-2-3 et C1), C.

\section{POACEAE}

Aegilops neglecta Req. ex Bertol. Th. (O1-2-3, A12, K1-2-3, C1, H1-2, Hd et AS1-2-3), C.

Aira cupaniana Guss. Th., (O1-2-3, A1-2, K1-2-3 et C1), AC.

Ampelodesma mauritanica (Poir.) T. Durand\& Schinz H. (O1-2-3, A1-2, K1-2-3, C1 et AS23), C.

Aristida caerulescens Desf. H., (O1-2-3, A1-2, C1, H1-2, Hd, AS1-2-3, SS1-2 et SC ), AC.

Avena barbata Pott ex Link Th. (O1-2-3, A1-2, K1-2-3, C1, H1-2, Hd et AS1-2-3), C.

Brachypodium distachyon (L.) P. Beauv. Th., (O1-2-3, A1-2, K1-2-3, C1, H1-2, Hd, AS1-2-3 et SS1-2), C.

Brachypodium retusum (Pers) P. Beauv. H. (O1-2-3, A1-2, K2 et C1), C.

Brachypodium sylvaticum (Huds.) P. Beauv. H. (O1-2-3, A1-2, K1-2-3 et C1), R.

Briza maxima L. Th., (O1-2-3, A1-2, K1-2-3 et C1), C.

Bromus hodeaceus subsp. divaricatus (Bonnier \& Layens) Kerguèlen Th., (O2 et A1)R.

Bromus madritensis subsp. madritensis $\mathrm{M}$. \& W. Th., (O1-2-3, A1-2, K1-2-3 et C1), C.

Bromus fasciculatus C. Presl Th., (O1-2), AC.

Bromus rubens L. Th., (O1-2-3, A1-2, K1-2-3, C1, H1-2, Hd, AS1-2-3, SS1-2, SC), C. 
Bromus scoparius L. Th., (O1-2-3, A1-2, K1-2-3 et $\mathrm{C} 1$ ), AC.

Bromus sterilis L. Th., (A2, K1-2 et AS1-2-3) AC.

Catapodium loliaceum (Huds.) Link Th., (O1-2-3, A1 et K1-2-3), AC.

Dactylis glomerata L. H., (O1-2-3, A1-2, K1-2-3, C1, H1-2, Hd, AS1-2-3), C.

Desmazeria rigida (L.) Tutin Th. (O1-2-3, A1-2, K1-2-3, C1, H1-2, Hd et AS1-2-3), C.

Festuca atlantica Duval-Jouve Th., (A2, K1-2, C1, AS3), [AM], R.

Festuca coerulescens Desf. H.(O1-2-3, A1-2, K12-3, C1 et AS1-2-3), C.

Hordeum murinum subsp. leporinum (Link) Arcang. Th. (O1-2-3, A1-2, K1-2-3, C1, H1-2, Hd, AS1-2-3 et SS1-2), C.

Hyparrhenia hirta (L.) Stapf. H., (O1-2-3, A1-2, K1-2-3, C1, H1-2, Hd, AS1-2-3 et SC), C.

Koeleria vallesiana (Honck.) Bert H. (O1-2-3, A1-2, K1-2-3, C1, H1-2, Hd et AS1-2-3),C.

Lagurus ovatus L. Th., (O1-2-3, A1-2, K1-2-3, C1, H1-2, Hd et AS1-2-3), C.

Lamarckia aurea (L.) Moench Th., (O1-2-3, A1-2, $\mathrm{K} 1-2-3$ et $\mathrm{C} 1$ ), $\mathrm{C}$.

Lygeum spartum L. H. (O1-2-3 C1, H1-2, Hd, AS12-3, SS1-2), C.

Pholiurus incurvus (L.) C.E.Hubb. Th., (O1-2-3, A1-2, K1-2-3, C1, H1-2, Hd, AS1-2-3 et SS1-2 AC.

Piptatherum miliaceum (L.) Coss. H., (O1-2-3, A1-2, K1-2-3, C1, H1-2, Hd, AS1-2-3, SS1-2,
SC et SM), C.

Rostraria balansae (Coss. \& Durieu) Holub Th., (O1), [AM.], R.

Stipa lagascae Roem. \& Schult. H., (O1-3, H1-2 et AS1-2-3), [AfnI], AC.

Stipa tenacissima L. H. (O1-2-3, A2, C1, H1-2, Hd et AS1-2-3), AC.

Vulpia membranacea (L.) Dumort. Th., (O1-2-3, A1-2, K1-2-3 et C1), C.

Vulpia myuros subsp. myuros Th. (O1-2-3, A1-2, K1-2-3 et C1), C.

\section{RUSCACEAE}

Ruscus hypophyllum L. H. (O1-2-3, A1-2, K1-2-3 et $\mathrm{C} 1$ ), $\mathrm{C}$.

\section{SMILACACEAE}

Smilax aspera L. Phl., (O1-2-3, A1-2, K1-2-3 et C1), C.

\section{DISCUSSION ET CONCLUSION}

La flore forestière des monts des Trara est constituée de 558 unités taxonomiques dont 503 espèces, 54 sous-espèces et 1 variété, appartenant à 87 familles et 306 genres. Les angiospermes dicotylédones forment le groupe systématique le plus important avec 446

\begin{tabular}{|c|c|c|c|}
\hline Taxon & Type biologique & Type biogéographique & Rareté \\
\hline Filago duriaei Coss. ex Lange & Th. & AMI. & R. \\
\hline Brassica maurorum Durieu & Th. & AM. & $\mathrm{R}$. \\
\hline Halimium umbellatum subsp. viscosum Willk. & Ch. & AMI. & R. \\
\hline Sedum maireanum Sennen & Ch. & AMI. & $\mathrm{R}$. \\
\hline Teucrium maghrebinum Greuter \& Burdet & Th. & AM. & R. \\
\hline Astragalus longidentatus Chater & Th. & AMI. & $\mathrm{R}$. \\
\hline Hammatolobium Kremerianum (Coss.) C. Muell. & $\mathrm{Ch}$. & AM. & $\mathrm{R}$. \\
\hline Orobanche leptantha Pomel & G. & $\mathrm{AM}$ & $\mathrm{R}$. \\
\hline Galium bourgeanum Coss. & Ch. & AM. & $\mathrm{R}$. \\
\hline Antirrhinum cirrhigerum (Welw. ex Ficalho) Rothm. & Ch. & AM. & $\mathrm{R}$. \\
\hline Ophrys dyris Maire & G. & AMI. & $\mathrm{R}$. \\
\hline Rostraria balansae (Coss. \& Durieu) Holub & Th. & AM. & $\mathrm{R}$. \\
\hline
\end{tabular}

Tableau 1: Taxons endémiques et rares des monts des Traras 
taxons appartenant à 59 familles et 240 genres; les monocotylédones contiennent 91 unités taxonomiques réparties dans 18 familles et 54 genres. Les ptéridophytes et les gymnospermes comptent 10 familles, 12 genres et 20 taxons.

Les familles des Leguminosae et des Asteraceae sont les plus représentées respectivement avec 79, 75 taxons, suivies par les Poaceae avec 33 taxons, les Umbelliferae 28, les Lamiaceae 26, les Brassicaceae 21, les Cistaceae 19, les Caryophyllaceae, 19 et les Orchidaceae 16. Le genre le plus riche en espèces est Ononis avec 10 taxons, suivi par Centaurea 9, Cistus et Ophrys 8, Silene, Euphorbia, Astragalus, Trifolium et Vicia avec 7 taxons.

Le spectre biologique est typique de l'ambiance bioclimatique méditerranéenne semi-aride, avec un important pourcentage pour les thérophytes $(42,40 \%), 12,26 \%$ pour les géophytes, $16,91 \%$ pour les hémicryptophytes, $9,37 \%$ pour les chaméphytes, $14,22 \%$ pour les nanophanérophytes et $4,84 \%$ pour les phanérophytes. Les Monts des Trara comptent 174 endémiques dont 41 endémiques Ibéro-algéromarocains, 37 endémiques algéro-marocains, 17 endémiques d'Afrique du Nord et 15 endémique ibéro-Nord Africains. De point de vue rareté; 36 taxons sont très rares, 49 taxons sont rares et 27 sont assez rares.

\section{Sur le plan chorologique, on souligne:}

1. la découverte de 3 nouveaux taxons pour l'Algérie: Ophrys dyris considérée comme spéciale au Maroc dans le catalogue de Jahandiez et Maire (1931) et une endémique ibéro-marocaine par Delforge (2001); Sedum maireanum endémique ibéro-marocaine (Fennane et al., 1999) et Antirrhinum cirrhigerum endémique ibéro-marocaine (Fennane et al. 2007).

9 Taxons nouveaux pour l'Oranie, il s'agit de: Asplenium hemionitis fougère très rares en Algérie dont une seule station est connue, et probablement complètement détruite dans le massif littoral de Bouzaréa à Alger. Cette nouvelle station est actuellement la seule en
Algérie qui abrite l'espèce. Les autres taxons sont présents en Algérie dans un ou plusieurs secteurs mais jamais signalés en Oranie, il s'agit de: Festuca atlantica, Bromus sterilis, Asphodelus ayardii, Arenaria leptoclados, Herniaria hemistemon, Blackstonia perfoliata subsp. perfoliata, Exaculum pusillum et Ajuga reptans.

Les nombres de taxons nouveaux pour les Trara s'élève à 16. Ces taxons sont présents en Oranie mais ils sont très rares et localisés, il s'agit de: Asplenium obovatum, Centranthus macrosiphon, Cytisus villosus, Daucus durieua, Filago duriaei, Filago heterantha subsp. dichotoma, Gagea algeriensis, Galium bourgeanum, Galium verticillatum, Halimium umbellatum subsp. viscosum, Lathyrus tingitanus, Limonium ferulaceum, Retama sphaerocarpa, Thymbra captitata, Thymelea argentata, et Wahlenbergia lobelioides subsp. nutabunda.

Astragalus longidentatus, Hammatolobium Kremerianum, Limonium asparagoides, Lithodora prostata subsp. lusitanica, Rostraria balansae et Teucrium maghrebinum sont des taxons trés rares, spécifiques aux Trara, ils n'existent en aucun autre endroit en Algérie.

Les monts des Trara, constituent un refuge pour de nombreuses espèces menacées citées dans la loi 1993 fixant la liste des espèces protégées en Algérie et mentionnées dans les travaux de nombreux auteurs pour le Maroc (Fennane \& Ibn Tattou, 1998) ou pour l'Algérie (Quézel et Santa, 1962). Le tableau 1 donne une liste succincte de taxons que nous jugeons utile de prendre en considération pour le classement de cette zone comme aire protégée (Medjahdi et al., 2008).

\section{BIBLIOGRAPHIE}

ALCARAZ, C. -1982- La végétation de l'Ouest algérien. Thèse d'état, Université de Perpignan, $415 \mathrm{p}$.

DELFORGE, P. -2001-Guide des Orchidées d'Europe, d'Afrique du Nord et du Proche- 
Orient. Ed. Delachaux et Niestlé, Paris. 640 p. FENNANE, M. \& M. IBN TATTOU -1998- Catalogue des plantes vasculaires rares, menacées ou endémiques du Maroc. Bocconea 8. Palerme. $243 \mathrm{p}$.

FENNANE, M., M. IBN TATTOU, M. J. MATHEZ, A. OUYAHYA, \& J. El OUALIDI (Ed.) -1999Flore pratique du Maroc. Vol. I, Institut Scientifique, Université Mohammed V, Rabat.

FENNANE, M., M. IBN TATTOU, A. OUYAHYA, \& EL OUALIDI J. (Ed.) -2007- Flore pratique du Maroc. Vol II, Institut Scientifique, Université Mohammed V, Rabat.

HADJADJ, S. -1995 - Les peuplements du Thuya de berbérie (Tetraclinis articulata (Vahl) Master) en Algérie: phytoécologie, syntaxonomie et potentialités sylvicoles. Thèse D'état, Université Aix-Marseille III, $159 \mathrm{p}$.

JAHANDIEZ, E. \& R. MAIRE -1931- Catalogue des Plantes du Maroc (Spermatophytes et Ptéridophytes). Tome I (Ptéridophytes, Gymnospermes et Monocotylédones), Imp. Minerva, Alger. $159 \mathrm{p}$.
MEDJAHDI B. -2001- Réponse de la végétation du littoral des monts des Trara (Ouest algérien) aux différents facteurs de dégradation. Mém. Magistère: Univ. De Tlemcen. 107 p. et Annexes.

MEDJAHDI B., A. MEDJAHDI, Kh. BOUAMAEUR \& Kh. BENABEDLI -2008- Plaidoyer pour la création d'une aire protégée et conservation des formations végétales contre les perturbations: cas des monts des Trara. Annales de l'INRGREF. № 12, 232-241 pp.

MEZIANI K. \& S. BELGATS -1984- Le cordon $d u$ naire littoral de la région de Mostaganem. Thèse D'état, Université Aix-Marseille III, 200 p.

QUEZEL, P. \& S. SANTA -1962- Nouvelle flore de l'Algérie et des régions désertiques méridionales. Tome I et Tome II. CNRS, Paris, 1087 p.

VALDÉS, B., M. REJDALI, A. A. E. KADMIRI, S. L. JURY \& J. M. MONTSERRAT -2002- Catalogue des plantes vasculaires du Nord du Maroc incluant des clés d'ídentification. Vol. I \& II, Consejo Superior de Investigaciones Científicas, Madrid. 1007p. 